\title{
Hydrogen states in hydrogen-passivated semiconducting barium disilicide measured via muon spin rotation
}

Zhihao $\mathrm{Xu}^{1}$, Takuma Sato ${ }^{1,2}$, Jumpei Nakamura ${ }^{3}$, Akihiro Koda ${ }^{3,4}$, Koichiro Shimomura ${ }^{3,4}$, Andrew B. Filonov ${ }^{5}$, Dmitry B. Migas ${ }^{5,6}$, and Takashi Suemasu ${ }^{1, *}$

${ }^{1}$ Institute of Applied Physics, University of Tsukuba, Tsukuba, Ibaraki 305-8573, Japan

${ }^{2}$ Université Grenoble Alpes, CNRS, CEA, INAC-SyMMES, 38000 Grenoble, France

${ }^{3}$ Muon Science Division Institute of Materials Structure Science, High Energy Accelerator Research Organization (KEK) Tsukuba, Ibaraki 305-0801, Japan

${ }^{4}$ Department of Materials Structure Science, The Graduate University for Advanced Studies (Sokendai), Tsukuba, Ibaraki 305-0801, Japan

${ }^{5}$ Department of Micro- and Nanoelectronics, Belarusian State University of Informatics and Radioelectronics, P. Browki 6, 220013 Minsk, Belarus

${ }^{6}$ National Research Nuclear University MEPhI (Moscow Engineering Physics Institute), Kashirskoe shosse 31, 115409 Moscow, Russia

* Corresponding author at:

Institute of Applied Physics, Faculty of Pure and Applied Sciences, University of Tsukuba, Tsukuba, Ibaraki 305-8573, Japan

*Electronic mail: suemasu@bk.tsukuba.ac.jp 
Passivation of $\mathrm{BaSi}_{2}$ films is essentially important for their use in solar cell applications. We study the effect of atomic $\mathrm{H}$ on photoresponsivity enhancement of $0.5-\mu \mathrm{m}$-thick $\mathrm{BaSi}_{2}$ films. A H supply duration of 15 min enhances the photoresponsivity of the $\mathrm{BaSi}_{2}$ film by a factor of 10 . We also investigate the electronic states of $\mathrm{H}$ in $\mathrm{BaSi}_{2}$ via muon spin rotation. An implanted muon $\left(\mu^{+}\right)$beam binds electrons to form muonium $\left(\mu^{+} e^{-}\right)$. Their response to thermal activation shows that $\mu^{+} e^{-}$accompanies a shallow energy level of approximately $31 \mathrm{meV}$ below the conduction band minimum (CBM), indicating that atomic $\mathrm{H}$ also serves as an electronically active donor impurity in $\mathrm{BaSi}_{2}$. This result is in good agreement with ab initio studies showing that a localized half-filled peak appears approximately $40 \mathrm{meV}$ below the $\mathrm{CBM}$ if the first neighbors of the $\mathrm{H}$ atom are one $\mathrm{Si}$ atom and one $\mathrm{Ba}$ atom. 


\section{Introduction}

Thin-film solar cell materials such as copper indium gallium selenide ( $\mathrm{CuIn} 1-\mathrm{x} \mathrm{Ga}_{\mathrm{x}} \mathrm{Se}_{2}$, CIGS) and cadmium telluride (CdTe) have attracted increasing attention owing to their low cost and high conversion efficiencies $(\eta){ }^{1)}$ For solar cell applications, researchers have focused on semiconducting barium disilicide $\left(\mathrm{BaSi}_{2}\right)^{2,3)}$ among various candidate materials because of its excellent properties ${ }^{4,5)}$ such as a band gap of $1.3 \mathrm{eV}$, which is more suitable than that of crystalline $\mathrm{Si}(1.1 \mathrm{eV})$ for a single-junction solar cell; its large absorption coefficient $(\alpha)$ of $3 \times 10^{4} \mathrm{~cm}^{-1}$ at $1.5 \mathrm{eV}$, exceeding that of CIGS; ${ }^{6-9)}$ and a long bulk minority carrier lifetime of $14 \mu$ s owing to its inactive grain boundaries. ${ }^{10,11)} \mathrm{BaSi}_{2}$ bulk crystals ${ }^{12,13)}$ and thin films often show n-type conductivity regardless of different growth techniques such as molecular beam epitaxy (MBE), ${ }^{6,14,15)}$ sputtering, ${ }^{16)}$ and vacuum evaporation. ${ }^{17-19)}$ We have achieved a value of $\eta$ approaching $10 \%$ in $\mathrm{p}-\mathrm{BaSi}_{2} / \mathrm{n}$ $\mathrm{Si}$ heterojunction solar cells, ${ }^{20,21)}$ and recently demonstrated the operation of $\mathrm{BaSi}_{2}$ homojunction solar cells. ${ }^{22)}$ We are currently focusing on further improving the minoritycarrier properties of $\mathrm{BaSi}_{2}$ absorber layers grown via $\mathrm{MBE}$. We use photoresponsivity as a measure of minority-carrier properties that significantly affect $\eta$. Our challenge is forming $\mathrm{BaSi}_{2}$ films with a low defect density. The photoresponsivity, carrier type, and carrier concentration of $\mathrm{BaSi}_{2}$ films are very sensitive to the ratio of the $\mathrm{Ba}$ deposition rate to the $\mathrm{Si}$ deposition rate $\left(R_{\mathrm{Ba}} / R_{\mathrm{Si}}\right)$ during MBE. ${ }^{15)}$ Maximum photoresponsivity is achieved at around $R_{\mathrm{Ba}} / R_{\mathrm{Si}}=2.2$ and 1.2 for growth at $580{ }^{\circ} \mathrm{C}$ and $650{ }^{\circ} \mathrm{C}$, respectively. 15,23) Even such high-quality $\mathrm{BaSi}_{2}$ films contain defects on the order of $10^{13} \mathrm{~cm}^{-3}$, confirmed by deep-level transient spectroscopy and electron paramagnetic resonance $(\mathrm{EPR}){ }^{24,25)}$

According to first-principle calculation by Kumar et al., ${ }^{26)} \mathrm{Si}$ vacancies $\left(\mathrm{V}_{\mathrm{Si}}\right)$ are most likely to exist as point defects in $\mathrm{BaSi}_{2}$ regardless of Si-rich or poor conditions, and generate localized states within the band gap. Under these circumstances, we recently found by experiment that atomic hydrogen $(\mathrm{H})$ is very effective in significantly enhancing the photoresponsivity of $\mathrm{BaSi}_{2}$ films, ${ }^{27,28)}$ which can be explained by passivating $\mathrm{V}_{\mathrm{Si}}$ in $\mathrm{BaSi}_{2}$ by atomic $\mathrm{H}$. Ab initio calculations revealed that $\mathrm{H}$ passivation of the Si dangling bonds at $\mathrm{V}_{\mathrm{Si}}$ can markedly decrease trap concentration in $\mathrm{BaSi}_{2} \cdot{ }^{28)} \mathrm{H}$ passivation is commonly used to reduce defects in semiconductors such as GaAs, diamond, and $\mathrm{Si}^{29-33)}$ Muon spin rotation $(\mu \mathrm{SR})$ is a powerful technique for investigating the electronic structure 
of isolated $\mathrm{H}$ centers in materials, because the implanted positive muon can be regarded as a light radioisotope of a proton (the muon mass is $1 / 9$ of the proton mass). Therefore, $\mu \mathrm{SR}$ has been applied to various semiconductors such as $\mathrm{ZnO}, \mathrm{CdO}, \mathrm{TiO}_{2}, \mathrm{SnO}_{2}, \mathrm{CdS}, \mathrm{Si}$, and $\mathrm{GaN}$ via their muonium analogs. ${ }^{34-41)}$ However, there has been no report on the $\mathrm{H}$ states of $\mathrm{BaSi}_{2}$.

In $\mu \mathrm{SR}$, positively charged spin-polarized muons $\left(\mu^{+}\right)$are first implanted into the material. Then, the $\mu^{+}$binds electrons to form muonium $\left(\mu^{+} e^{-}\right)$; therefore, the local electronic structure of muon is virtually equivalent to that of $\mathrm{H}$ after a small correction due to a difference in the reduced mass. The spin motion of $\mu^{+}$is significantly affected by the captured electron or even the neighboring nucleus in the material, and gradually decays. The decay of the $\mu^{+}$into a positron $\left(e^{+}\right)$occurs after a mean lifetime of approximately $2.2 \mu \mathrm{s}$, and the emitted $e^{+}$provides information on the spin of the parent $\mu$ + . For example, $\mu \mathrm{SR}$ revealed that $\mathrm{H}$ atoms behave as shallow donors in $\mathrm{GaN},{ }^{36}$ ) explaining why high hole concentrations were difficult to achieve in magnesium-doped $\mathrm{GaN}$. This is because $\mathrm{H}$ is one of the most common impurities in semiconductors. In this study, we report on the observation of the paramagnetic muonium spectrum in $\mathrm{BaSi}_{2}$. The calculated muonium state has a hyperfine parameter equivalent to a magnetic field of 8.8 $\mathrm{mT}$. Furthermore, the energy level within the band gap of this muonium is estimated to be approximately $31 \mathrm{meV}$ below the conduction band minimum (CBM) of $\mathrm{BaSi}_{2}$, as determined from the measured activation energy for thermal ionization. We also discuss the position of $\mathrm{H}$ atom in the lattice of $\mathrm{BaSi}_{2}$ by first-principle calculations, and propose a model that well explains the above-mentioned shallow donor level.

\section{Methods}

We fabricated 0.5- $\mu$ m-thick $\mathrm{BaSi}_{2}$ epitaxial films on a low-resistivity $(\rho)$ Czochralski $(\mathrm{Cz}) \mathrm{n}^{+}-\mathrm{Si}(111)(\rho<0.01 \Omega \mathrm{cm})$ substrate via MBE. The 500-nm-thick $\mathrm{BaSi}_{2}$ films were irradiated with atomic $\mathrm{H}$ produced by a radio-frequency plasma generator for various irradiation durations $\left(t_{\mathrm{BaSi}: \mathrm{H}}\right)$ from 0 to $30 \mathrm{~min}$ at a substrate temperature $\left(T_{\mathrm{S}}\right)$ of $580{ }^{\circ} \mathrm{C}$. The vacuum level and power of the plasma generator were kept at $10^{-3} \mathrm{~Pa}$ and $10 \mathrm{~W}$, respectively. Finally, a 3-nm-thick amorphous Si layer was deposited at $T_{\mathrm{S}}=180{ }^{\circ} \mathrm{C}$ to prevent surface oxidation. ${ }^{42)}$ To measure photoresponsivity, we sputtered an $80 \mathrm{~nm}$-thick 
indium-tin-oxide (ITO) electrode on the front side and $150 \mathrm{~nm}$-thick Al electrodes on the back side. The photoresponsivity spectra were evaluated under a bias voltage of $0.3 \mathrm{~V}$ applied to the front ITO electrode with respect to backside electrodes through a lock-in technique using a xenon lamp with a 25-cm-focal-length single monochromator (Bunko Keiki, SM-1700A and RU-60N). The light intensity of the lamp was calibrated using a pyroelectric sensor (Melles Griot, 13PEM001/J). Photoluminescence (PL) measurements were carried out at $8 \mathrm{~K}$ by exciting the samples from the $\mathrm{BaSi}_{2}$ side. The excitation wavelength was $442 \mathrm{~nm}$. The PL was analyzed with another 25-cm-focal-length single monochromator, detected by a liquid-nitrogen-cooled $\mathrm{InP} / \mathrm{InGaAs}$ photomultiplier tube (Hamamatsu Photonics R5509-72) and amplified via the lock-in technique.

Conventional $\mu \mathrm{SR}$ experiments were performed using the ARTEMIS spectrometer installed in the MLF S1 Instrument of the Japan Proton Accelerator Research Complex (J-PARC). These experiments measured the time-dependent Mu- $e$ decay asymmetry $A(t)$ under a zero field (ZF), a longitudinal field (LF) in which the initial muon polarization $p(0)$ was parallel to the applied magnetic field $(B)$, and a weak transverse field (TF) in which $p(0)$ was perpendicular to $B$. A $100 \%$ spin-polarized pulsed $\mu^{+}$beam with a full width at half maximum of around $100 \mathrm{~ns}$ and momentum of $27 \mathrm{MeV} / c$ was used to irradiate a polycrystalline stoichiometric $\mathrm{BaSi}_{2}$ sputter target (Tosoh Corporation) loaded on a He-flow cryostat to monitor $A(t)$ over a temperature range from 6 to $300 \mathrm{~K}$.

In order to estimate possible energy positions of defect levels caused by atomic $\mathrm{H}$ in the interstitial sites in the $\mathrm{BaSi}_{2}$ bulk we have implemented the first principles total energy projector-augmented wave method (code VASP) ${ }^{43-45)}$ with plane wave basis set and the generalized gradient approximation of Perdew-Burke-Ernzerhof ${ }^{46)}$ for the exchange and correlation potentials. The supercell (the $2 \times 3 \times 2$ increased crystallographic unit cell of $\mathrm{BaSi}_{2}$ ) was used to model one atomic $\mathrm{H}$ as an interstitial impurity in $\mathrm{BaSi}_{2}$ (no $\mathrm{V}_{\mathrm{Si}}$ was considered) and to minimize a defect-defect interaction. Total energy minimization, via an optimization of lattice parameters and a relaxation of atomic positions in a conjugate gradient routine, was obtained by calculating the Hellmann-Feynman forces and the stress tensor. We set the energy cutoff at $325 \mathrm{eV}$. To perform integration over the Brillouin zone the $5 \times 5 \times 5$ mesh of Monkhorst-Park points was used and it was found to be sufficient to obtain the convergence in total energy to be less than $0.001 \mathrm{eV}$ per unit cell. The atomic relaxation was stopped when forces on the atoms were smaller than $0.05 \mathrm{eV} / \AA$. Total 
density of states (DOSs) has been calculated by the tetrahedron method with Blöchl corrections.

\section{Results and discussion}

Figure 1 shows the photoresponsivity spectra of the $0.5-\mu \mathrm{m}$-thick $\mathrm{BaSi}_{2}$ epitaxial films passivated for various atomic $\mathrm{H}$ supply durations $\left(t_{\mathrm{BaSi}: \mathrm{H}}\right)$. Because there was no built-in electric field in the $\mathrm{BaSi}_{2}$ films, we applied a bias voltage of $0.3 \mathrm{~V}$ to the frontsurface ITO electrode with respect to the rear Al electrode so that the photogenerated electrons were extracted to the front ITO electrode. The photoresponsivity sharply increased for photon energies greater than the band gap. We observed a maximum sample photoresponsivity of about $2.5 \mathrm{~A} / \mathrm{W}$ at a photon energy $(E)$ of $1.6 \mathrm{eV}$ at $t_{\mathrm{BaSi}: \mathrm{H}}=15 \mathrm{~min}$. This value is approximately one order of magnitude higher than the reference sample prepared without $\mathrm{H}$ supply. ${ }^{15)}$ At $t_{\mathrm{BaSi}: \mathrm{H}}=15$ min, the $\mathrm{H}$ concentration reached about $1 \times$ $10^{19} \mathrm{~cm}^{-3} .{ }^{28)}$ The photoresponsivity of the sample was almost the same for both $t_{\mathrm{BaSi}: \mathrm{H}}=$ $15 \mathrm{~min}$ and $t_{\mathrm{BaSi}: \mathrm{H}}=10 \mathrm{~min}$ at $E>2.5 \mathrm{eV}$, which was attributed to saturation of passivated defects on the top surface due to the large $\alpha$ of $\mathrm{BaSi}_{2}$. Furthermore, the photoresponsivity was decreased by either lengthening or shortening $t_{\mathrm{BaSi}: \mathrm{H}}$, and the optimum $t_{\mathrm{BaSi} \mathrm{H}}$ was found to be $15 \mathrm{~min}$. According to Ref. 28, the enhancement of photoresponsivity was explained by the increase of carrier lifetime due to the passivation of $\mathrm{V}_{\mathrm{Si}}$, which was 1 , 14, and $3 \mu \mathrm{s}$ at $t_{\mathrm{BaSi}: \mathrm{H}}=1,15$, and $30 \mathrm{~min}$, respectively. The $t_{\mathrm{BaSi}: \mathrm{H}}$ dependence of photoresponsivity is discussed later in relation to the $t_{\mathrm{BaSi}} \mathrm{H}$ dependence of PL intensity.

Figure 2(a) shows the PL spectra of these samples measured at $8 \mathrm{~K}$. The PL intensity significantly depended on $t_{\mathrm{BaSi}} \mathrm{H} . \mathrm{BaSi}_{2}$ is an indirect bandgap semiconductor; therefore, its PL can be ascribed to the radiative transition of electrons between defect states within the bandgap. In the reference sample $\left(t_{\mathrm{BaSi}: \mathrm{H}}=0 \mathrm{~min}\right)$, we observed weak and broad PL. This PL spectrum was reproducible by two Gaussian curves with peaks at $0.81 \mathrm{eV}$ and $0.99 \mathrm{eV}$ as shown in Fig. 2(b). The energy of $0.81 \mathrm{eV}$ is approximately $0.5 \mathrm{eV}$ smaller than the band gap of $\mathrm{BaSi}_{2}$, indicating the presence of deep defect levels and that the photogenerated carriers in the $\mathrm{BaSi}_{2}$ film recombined via such defect levels. As $t_{\mathrm{BaSi}: \mathrm{H}}$ increased to 1 and 5 min, the PL intensity becomes higher in Fig. 2(a). The PL spectra of these samples can also be fitted by two Gaussian curves in Fig. 2(b). As $t_{\mathrm{BaSi}: \mathrm{H}}$ increased much further, however, the PL intensity started to decrease. Note that the PL at around 
$\geq 5 \mathrm{~min}$. According to the Shockley-Read-Hall recombination model, ${ }^{47)}$ deep levels promote recombination of photogenerated carriers more than shallow levels. Therefore, we attribute the photoresponsivity enhancement in Fig. 1 to the reduction of deep defect levels in $\mathrm{BaSi}_{2}$ films by $\mathrm{H}$ passivation. We should not overlook the fact that the $t_{\mathrm{BaSi}} \mathrm{H}$ for the highest photoresponsivity was different from that for the highest PL. This difference is attributed to the difference in carrier recombination mechanism between the PL and photoreponsivity. In the case of photoreponsivity, carrier recombinations of any kind degrade the photoresponsivity. In contrast, the PL intensity decreases by nonradiative recombination. Therefore, we interpret the $t_{\mathrm{BaSi}} \mathrm{H}$ dependences of photoresponsivity and PL as follows. With increasing $t_{\mathrm{BaSi}} \mathrm{H}$ up to $5 \mathrm{~min}$, the nonradiative recombination rate decreased, and thus the PL intensity increased. For further increase of $t_{\mathrm{BaSi}: \mathrm{H}}$ up to $15 \mathrm{~min}$, the radiative recombination rate also decreased, leading to the enhancement of photoresponsivity and the decrease of PL. However, for much further increase of $t_{\mathrm{BaSi}} \mathrm{H}$, the nonradiative recombination rate started to increase, decreasing both the photoresponsivity and PL. At present, there is not enough evidence to trace the origin of defect levels. EPR studies are now under investigation for this purpose. ${ }^{25)}$

We move on to the results of $\mu \mathrm{SR}$. Figure 3(a) shows the $\mu \mathrm{SR}$ asymmetry time spectra obtained at 6,96 , and $300 \mathrm{~K}$ under a transverse field at $B_{\mathrm{TF}}=2 \mathrm{mT}$ for $\mathrm{BaSi}_{2}$. The implanted $\mu^{+}$ions are found to be in the diamagnetic state above $6 \mathrm{~K}$. The data points are thus fitted to

$$
A(t)=A_{0} \exp (-\lambda t) \cos \left(\omega_{\mu} t+\varphi\right) .
$$

Here, $A_{0}$ is a coefficient related to the amount of $\mu^{+}, \lambda$ is the damping coefficient, and $\omega_{\mu}$ is the $\mu^{+}$precession frequency given by $\gamma_{\mu} B$, where $\gamma_{\mu}$ is the muon gyromagnetic ratio equal to $2 \pi \times 135.5 \mathrm{MHz} / \mathrm{T}$; $t$ is the time; and $\varphi$ is the phase of the corresponding decay positron detector. The activation energy $E_{\mathrm{a}}$ for $\mu$ ionization was found to be approximately $31 \mathrm{meV}$ by fitting the fractional yield in Fig. 3(b) with the function $\beta_{1}+\beta_{2} \exp \left(-E_{a} / k_{\mathrm{B}} T\right)$ over the temperature range from 71 to $196 \mathrm{~K}$. Here, $\beta_{1}$ and $\beta_{2}$ are coefficients, $k_{\mathrm{B}}$ is the Boltzmann constant, and $T$ is the absolute temperature. This activation energy represents a direct transition from the defect level to the CBM. This result suggests that atomic $\mathrm{H}$ in 
$\mathrm{BaSi}_{2}$ acts as a shallow donor and would contribute to the unintentional n-type conductivity of $\mathrm{BaSi}_{2}$. On the basis of these results, the reason for the marked enhancement of photoresponsivity by the atomic $\mathrm{H}$ supply is considered as follows. Deep defect levels due to $\mathrm{V}_{\mathrm{Si}}$ are filled by electrons donated by the shallow donor level of atomic $\mathrm{H}$. Therefore, they no longer can accept photogenerated electrons, meaning that these deep levels are made inactivated by atomic $\mathrm{H}$. When the $\mathrm{H}$ content increases further, the electron concentration (majority carriers) increases, leading to the reduction in photoresponsivity. In line with this assumption, the measured charge carrier concentrations and Hall mobilities of $0.5-\mu \mathrm{m}$-thick $\mathrm{BaSi}_{2}$ layers at room temperature are $p$-type, $p=1.1 \times 10^{17} \mathrm{~cm}^{-3}$, hole mobility $\mu_{p}=25 \mathrm{~cm}^{2} / \mathrm{Vs}\left(t_{\mathrm{BaSi}: \mathrm{H}}=1 \mathrm{~min}\right), p$-type, $p=1.8$ $\times 10^{17} \mathrm{~cm}^{-3}, \mu_{p}=97 \mathrm{~cm}^{2} / \mathrm{Vs}\left(t_{\mathrm{BaSi}: \mathrm{H}}=15 \mathrm{~min}\right)$, and $n$-type, $n=9 \times 10^{15} \mathrm{~cm}^{-3}$, electron mobility $\mu_{n}=1240 \mathrm{~cm}^{2} / \mathrm{Vs}\left(t_{\mathrm{BaSi}: \mathrm{H}}=30 \mathrm{~min}\right)$. These results reveal that the type of majority carriers changed from holes to electrons as $t_{\mathrm{BaSi}} \mathrm{H}$ was increased from 1 to $30 \mathrm{~min}$.

In our previous paper, ${ }^{28)}$ we attribute the enhancement of photoresponsivity to the increase of carrier lifetime by atomic $\mathrm{H}$ passivation of $\mathrm{V}_{\mathrm{Si}}$, meaning that $\mathrm{H}$ atoms saturate dangling bonds at $\mathrm{V}_{\mathrm{Si}}$. However, it is hard to expect that all atomic $\mathrm{H}$ are involved to neutralize Si dangling bands caused by the presence of $\mathrm{V}_{\mathrm{Si}}$, we have also investigated possible changes in the $\mathrm{BaSi}_{2}$ energy spectrum if atomic $\mathrm{H}$ is present as an interstitial impurity. This issue has been earlier studied by us ${ }^{28)}$ identifying two distinct cases. Thus, if the first neighbors of a $\mathrm{H}$ atom are two $\mathrm{Ba}$ atoms, $\mathrm{BaSi}_{2}$ behaves as a degenerate semiconductor because the Fermi level $\left(E_{\mathrm{F}}\right)$ crosses the bottom of the conduction band (see Fig. 4(a)). It is similar to the situation when one electron is added to the system. The second case occurs if the first neighbors of a $\mathrm{H}$ atom are two $\mathrm{Si}$ and two $\mathrm{Ba}$ atoms providing a localized peak in the energy spectrum (one band) located close to the middle of the gap in the corresponding DOS (see Fig. 4 (b)). This peak can be viewed as a halffilled state and it is due to the $\mathrm{H}-s, \mathrm{Si}-s, \mathrm{Si}-p$ states ( $\mathrm{Si}$ atoms belong to the same tetrahedron with two $\mathrm{Si}$ atoms bonded to $\mathrm{H}$ ). In addition to that we could detect the third case if the first neighbors of a $\mathrm{H}$ atom were one $\mathrm{Si}$ atom and one $\mathrm{Ba}$ atom. Here a localized half-filled state (one band) appears approximately $40 \mathrm{meV}$ below the bottom of the conduction band (see Fig. 4 (c)), which can be treated as a shallow donor level in perfect agreement with our experimental data. We have identified the $\mathrm{H}-s, \mathrm{Ba}-d, \mathrm{Si}-s, \mathrm{Si}-p$ states ( $\mathrm{Si}$ atoms belong to the same tetrahedron with one $\mathrm{Si}$ atom bonded to $\mathrm{H}$ and $\mathrm{Ba}$ atom is 
the neighbor to $\mathrm{H}$ atom) to define this shallow donor level. Moreover, it is necessary to point out here, the most stable case in the total energy is the one with the shallow donor level (presented in Fig. 4(c)), while the least stable case - a degenerate semiconductor (presented in Fig. 4(a)).

Figure 5(a) shows the $\mu \mathrm{SR}$ spectra at $50 \mathrm{~K}$ under different longitudinal external magnetic fields of $0-35 \mathrm{mT}$. The increase of $A(0)$ from $4 \%$ to approximately $20 \%$ as $B$ increases from 0 to $35 \mathrm{mT}$ clearly indicates that a fraction of $\mu^{+}$forms a paramagnetic state. Under the LF, wherein $B$ was applied parallel to the initial spin polarization, the time evolution of $\mu^{+}$spin polarization for the muonium-like paramagnetic states $p(t, b)$ is described by that of the spin-triple $(F=1)$ and spin-singlet $(F=0)$ states, and is given by, ${ }^{48)}$

$$
p(t, b)=\frac{1}{2\left(1+b^{2}\right)}\left[\left(1+2 b^{2}\right)+\cos \omega_{\mathrm{b}} t\right]
$$

where $\omega_{\mathrm{b}}$ is the muon spin precession frequency for the $F=0$ state and $b=B / B_{\mathrm{C}}$, and $B_{\mathrm{C}}$ is the effective magnetic field corresponding to the magnitude of the hyperfine parameter. The second term in Eq. (2) can be averaged to zero. This is because $\omega_{\mathrm{b}}$ exceeds the limit determined by the experimental time resolution (the Nyquist frequency for the time resolution of $100 \mathrm{~nm}$ at J-PARC MUSE is $5 \mathrm{MHz}$ ). The residual polarization (= $1 / 2$ for $B$ $=0$ ) therefore corresponds to the $F=1$ state. As shown in Fig. 5(b), the experimental data are reproduced well by setting $B_{\mathrm{C}}$ at $8.8 \mathrm{mT}$. This value is equivalent to a frequency of $246 \mathrm{MHz}$ and is about $1 / 18$ that in vacuum $(4.46 \mathrm{GHz})$. From this value we estimate the

Bohr radius in $\mathrm{BaSi}_{2}$ to be increased by $(18)^{1 / 3} \sim 2.6$ via charge screening. This value agrees relatively well with that calculated using the effective electron mass of $m_{\mathrm{e}}=0.4 m_{0}\left(m_{0}\right.$ is the free electron mass) and dielectric constant $\left(\varepsilon_{\mathrm{r}}=14\right)$ of $\left.\mathrm{BaSi}_{2}{ }^{49}\right)$ in a simple hydrogenatom model.

\section{Conclusion}

We have demonstrated the highest photoresponsivity ever reported in 500-nm-thick $\mathrm{BaSi}_{2}$ films passivated with atomic $\mathrm{H}$. This directly verifies the contribution of atomic $\mathrm{H}$ to improving minority-carrier properties of $\mathrm{BaSi}_{2}$ thin films. A paramagnetic muonium 
center $\left(\mu^{+} e^{-}\right)$was confirmed in $\mathrm{BaSi}_{2}$ by $\mu \mathrm{SR}$. The temperature dependence of the yield for this state shows that $\mathrm{H}$ in $\mathrm{BaSi}_{2}$ acts as a shallow donor with an activation energy of approximately $31 \mathrm{meV}$. The presence of such a shallow donor level was verified by $a b$ initio studies when a $\mathrm{H}$ atom was located at an interstitial site and its first neighbors were one $\mathrm{Si}$ atom and one $\mathrm{Ba}$ atom. The hyperfine parameter obtained is approximately 246 $\mathrm{MHz}(8.8 \mathrm{mT})$.

\section{Acknowledgements}

We thank the staff of J-PARC MUSE for their technical support during the $\mu$ SR experiment. The muon experiment was supported by the Inter-University Research Program of the Institute for Materials Structure Science of Japan (IMSS) and the High Energy Accelerator Research Organization (KEK) (Proposal No. 2018B0045). This work was financially supported by a Grant-in-Aid for Scientific Research A (18H03767) from the Japan Society for the Promotion of Science (JSPS) and by the Belarusian National Research Programs "Convergence 2020," "Materials Science, New Materials and Technology" from the Belarusian Republican Foundation for Fundamental Research (Grant No. F18MC-012). D.B. Migas also acknowledges the partial financial support of the "Improving of the Competitiveness" Program of the National Research Nuclear University MEPhI - Moscow Engineering Physics Institute. 


\section{References}

1) T. D. Lee and A. U. Ebong, Renew. Sust. Energ. Rev. 70, 1286 (2017).

2) J. Evers J, G. Oehlinger, and A. Weiss, Angew. Chem., Int. Ed. Engl. 16659 (1977).

3) M. Imai and T. Hirano, Phys. Rev. B 5811922 (1998).

4) T. Suemasu, Jpn. J. Appl. Phys. 54, 07JA01 (2015).

5) T. Suemasu and N. Usami, J. Phys. D. Appl. Phys. 50, 023001 (2017).

6) K. Morita, Y. Inomata, and T. Suemasu, Thin Solid Films 508, 363 (2006).

7) D. B. Migas, V. L. Shaposhnikov, and V. E. Borisenko, Phys. Status Solidi (b) 244, 2611 (2007).

8) K. Toh, T. Saito, and T. Suemasu, Jpn. J. Appl. Phys. 50, 068001 (2011).

9) M. Kumar, N. Umezawa, and M. Imai, Appl. Phys. Express 7, 071203 (2014).

10) K. O. Hara, N. Usami, K. Nakamura, R. Takabe, M. Baba, K. Toko, and T. Suemasu, Appl. Phys. Express 6, 112302 (2013).

11) M. Baba, M. Kohyama, and T. Suemasu, J. Appl. Phys. 120, 085311 (2016).

12) T. Nakamura, T. Suemasu, K. Takakura, F. Hasegawa, A. Wakahara, and M. Imai, Appl. Phys. Lett. 81, 1032 (2002).

13) S. Kishino, T. Imai, T. Iida, Y. Nakaishi, M. Shinada, Y. Takanashi, and N. Hamada, J. Alloys Compd. 428, 22 (2007).

14) W. Du, M. Baba, K. Toko, K. O. Hara, K. Watanabe, T. Sekiguchi, N. Usami, and T. Suemasu, T. J. Appl. Phys. 115, 223701 (2014).

15) R. Takabe, T. Deng, K. Kodama, Y. Yamashita, T. Sato, K. Toko, and T. Suemasu, J. Appl. Phys. 123, 045703 (2018).

16) S. Matsuno, R. Takabe, S. Yokoyama, K. Toko, M. Mesuda, H. Kuramochi, and T. Suemasu, Appl. Phys Express 11, 071401 (2018).

17) K. O. Hara, J. Yamanaka, K. Arimoto, K. Nakagawa, T. Suemasu, and N. Usami, Thin Solid Films 595, 68 (2015).

18) K. O. Hara, C. T. Trinh, K. Arimoto, J. Yamanaka, K. Nakagawa, Y. Kurokawa, T. Suemasu, and N. Usami, J. Appl. Phys. 120, 045103 (2016).

19) C. T. Trinh, Y. Nakagawa, K. O. Hara, R. Takabe, T. Suemasu, and N. Usami, Mater. Res. Express 3, 076204 (2016).

20) S. Yachi, R. Takabe, H. Takeuchi, K. Toko, and T. Suemasu, Appl. Phys. Lett. 109, 072103 (2016). 
21) T. Deng, T. Sato, Z. Xu, R. Takabe, S. Yachi, Y. Yamashita, K. Toko, and T. Suemasu, Appl. Phys. Express 11, 062301 (2018).

22) K. Kodama, Y. Yamashita, K. Toko, and T. Suemasu, Appl. Phys. Express 12, 041005 (2019).

23) Y. Yamashita, Y. Takahara, T. Sato, K. Toko, A. Uedono, and T. Suemasu, Appl. Phys. Express 12, 055506 (2019).

24) Y. Yamashita, T. Sato, M. Emha Bayu, K. Toko, and T. Suemasu, Jpn. J. Appl. Phys. 57, 075801 (2018).

25) T. Sato, C. Lombard, Y. Yamashita, Z. Xu, L. Benincasa, S. Gambarelli, and T. Suemasu, Appl. Phys. Express 12, 061005 (2019).

26) M. Kumar, N. Umezawa, W. Zou, and M. Imai, J. Mater. Chem. A 5, 25293 (2017).

27) Z. Xu, Z. Xu, K. Gotoh, T. Deng, T. Sato, R. Takabe, K. Toko, N. Usami, and T. Suemasu, AIP Adv. 8, 055306 (2018).

28) Z. Xu, D. A. Shohonov, A. B. Filonov, K. Gotoh, T. Deng, S. Honda, K. Toko, N. Usami, D. B. Migas, V. E. Borisenko, and T. Suemasu, Phys. Rev. Mater. 3, 065403 (2019).

29) M. I. Landstrass and K. V. Ravi, Appl. Phys. Lett. 55, 1391 (1989).

30) N. M. Johnson, R. D. Burnham, R. A. Street, and R. L. Thornton, Phys. Rev. B 33, 2 (1986).

31) J. L. Benton, C. J. Doherty, S. D. Ferris, D. L. Flamm, L. C. Kimerling, and H. J. Leamy, Appl. Phys. Lett. 36, 670 (1980).

32) M. Taguchi, A. Terakawa, E. Maruyana, and M. Tanaka, Prog. Photovol.: Res. Appl. 13, 481 (2005).

33) Y. Tsunomura, Y. Yoshimine, M. Taguchi, T. Baba, T. Kinoshita, H. Kanno, H. Sakata, E. Maruyama, and M. Tanaka, Sol. Energ. Mater. Sol. Cells 93, 670 (2009).

34) S. F. J. Cox, E. A. Davis, and P. J. C. King, J. M. Gil, H. V. Alerto, R. C. Vilao, J. P. Duarte, N. A. de Campos, and R. L. Lichit, J. Phys-Condensed Matter 13, 9001 (2001).

35) D. G. Eshchenko, V. G. Storchak, S. P. Cottrell, and S. F. J. Cox, Phys. Rev. B 7, 073201 (2003).

36) K. Shimomura, R. Kadono, K. Ohishi, M. Mizuta, M. Saito, K. H. Chow, B. Hitti, and R. L. Lichiti, Phys. Rev. Lett. 92135505 (2004).

37) H. V. Alberto, J. P. Duarte, A. Weidinger, R. C. Vilao, J. M. Gil, N. A. de Campos, 
K. Fostiropouios, T. Prokscha, A. Sueter, and E. Morenzoni, Physca B 404, 870 (2009).

38) H. V. Alberto, A. Weidinger, R. C. Vilao, J. P. Duarte, J. M. Gim, J. S. Lord, and S. F. J. Cox, Phys. Rev. B 24, 245205 (2010).

39) B. B. Baker, Y. G. Celebi, R. L. Lichti, H. N. Bani-Salameh, P. W. Mengyan, B. R. Carroll, Physics Procedia 30, 101 (2012).

40) H. V. Alberto, R. C. Vilao, J. P. Duarte, J. M. Gil, A. Weidinger, J. S. Lord, and S. F. J. Cox, Phys. Rev. B 3, 035203 (2012).

41) T. Prokscha, H. Luetkens, E. Morenzoni, G. J. Nieuwenhuys, A. Suter, M. Dobeli, M. Horisberger, and E. Pomjakushna, Phys. Rev. B 90, 235303 (2014).

42) R. Takabe, K. O. Hara, M. Baba, W. Du, N. Shimada, K. Toko, N. Usami, and T. Suemasu, J. Appl. Phys. 115, 193510 (2014).

43) G. Kresse and J. Hafner, Phys. Rev. B 49, 14251 (1994).

44) G. Kresse and J. Furthmüller, Phys. Rev. B 54, 11169 (1996).

45) G. Kresse and J. Joubert, Phys. Rev. B 59, 1758 (1999).

46) P. Perdew, S. Burke, and M. Ernzerhof, Phys. Rev. Lett. 77, 3865 (1996).

47) W. Shockley and W. T. Read, Jr., Phys. Rev. 87, 835 (1952).

48) H. Okabe, M. Hiraishi, S. Takeshita, A. Koda, K. M. Kojima, and R. Kadono, Phys. Rev. B 98, 075210 (2018).

49) D. B. Migas, V. L. Shaposhnikov, and V. E. Borisenko, Phys. Status Solidi B 244, 2611 (2007). 


\section{Figure captions}

Figure 1: Photoresponse spectra of $0.5-\mu$ m-thick $\mathrm{BaSi}_{2}$ layers passivated by atomic $\mathrm{H}$ with various $t_{\mathrm{BaSi}: \mathrm{H}}$ values in the range $0-30 \mathrm{~min}$.

Figure 2: (a) PL spectra of $0.5-\mu \mathrm{m}$-thick $\mathrm{BaSi}_{2}$ layers at $8 \mathrm{~K}$, passivated by atomic $\mathrm{H}$ with various $t_{\mathrm{BaSi}: \mathrm{H}}$ values in the range $0-30 \mathrm{~min}$. (b) PL spectra of samples at $t_{\mathrm{BaSi}: \mathrm{H}}=0,1$, and 5 min fitted using two Gaussian curves.

Figure 3: (a) Asymmetric $\mu \mathrm{SR}$ time spectra in $\mathrm{BaSi}_{2}$ at $B_{\mathrm{TF}}=2 \mathrm{mT}$ measured at 6,96 , and 300 K. (b) Fractional yield of a diamagnetic state against temperature. The solid curve is the fitting result.

Figure 4: Total DOS of $\mathrm{BaSi}_{2}$ with atomic $\mathrm{H}$ acting as an interstitial impurity. (a) indicates the case when the first neighbors of a $\mathrm{H}$ atom are two $\mathrm{Ba}$ atoms with interatomic distance of $2.62 \AA$, (b) when the first neighbors of a $\mathrm{H}$ atom are two $\mathrm{Si}$ atoms (interatomic distance of $1.96 \AA$ ) and two Ba atoms (interatomic distance of $2.82 \AA$ ), and (c) when the first neighbors of a $\mathrm{H}$ atom are one $\mathrm{Si}$ atom (interatomic distance of $1.55 \AA$ ) and one $\mathrm{Ba}$ atom (interatomic distance of $2.63 \AA$ ). Zero at the energy scale corresponds to the top of the valence band. The vertical dashed lines indicate the Fermi level. Atomic configurations in the vicinity of the $\mathrm{H}$ atom is also shown. The large and green spheres indicate Ba atoms, medium and blue spheres stand for Si atoms and $\mathrm{H}$ atoms are represented by small grey spheres, respectively.

Figure 5: (a) Asymmetric $\mu \mathrm{SR}$ time spectra at $B_{\mathrm{LF}}=0,5$, and $35 \mathrm{mT}$ measured at $50 \mathrm{~K}$. The data points are experimentally obtained, and the curves are the fitting results. (b) Initial asymmetry against $B_{\mathrm{LF}}$ and its fitting curve at $50 \mathrm{~K}$. The magnetic field corresponding to the hyperfine parameter is estimated to be $8.8 \mathrm{mT}$. 


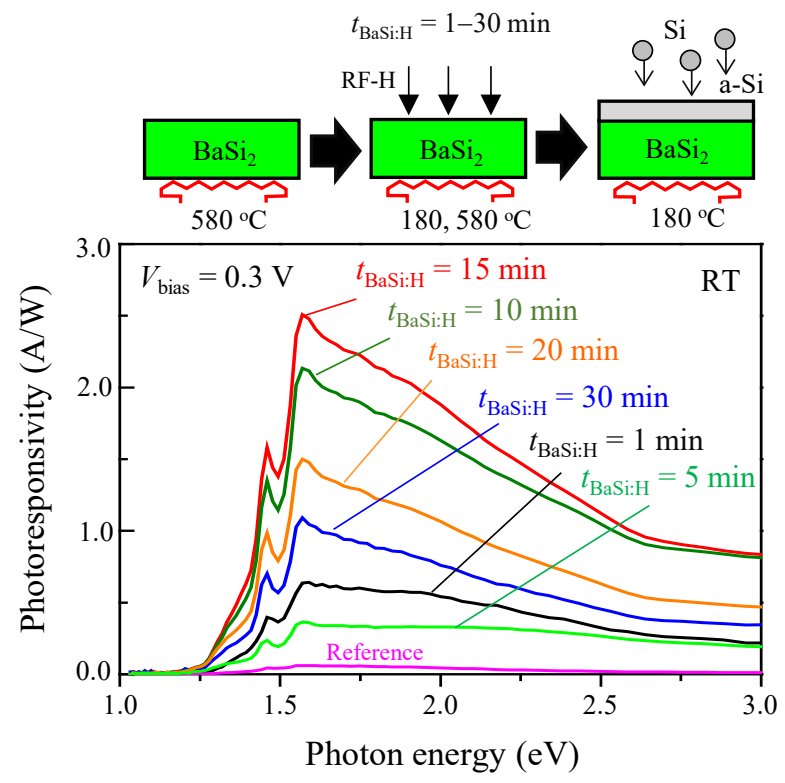

Fig. 1 

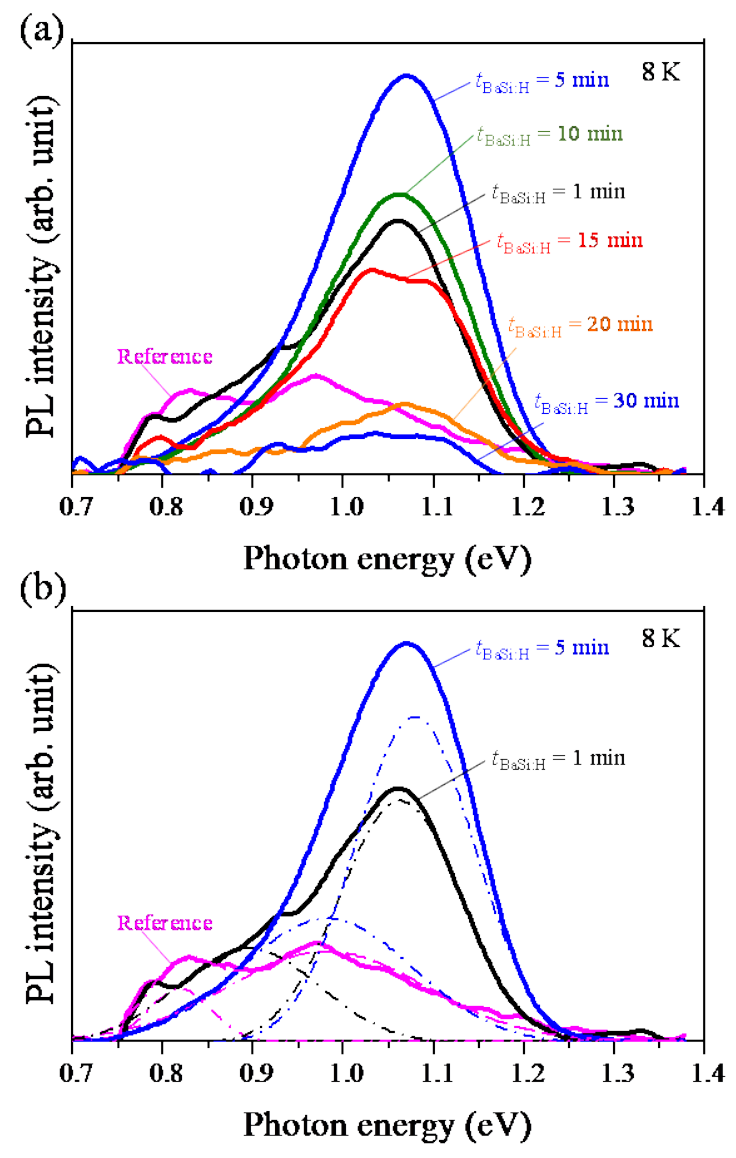

Fig. 2 
(a)
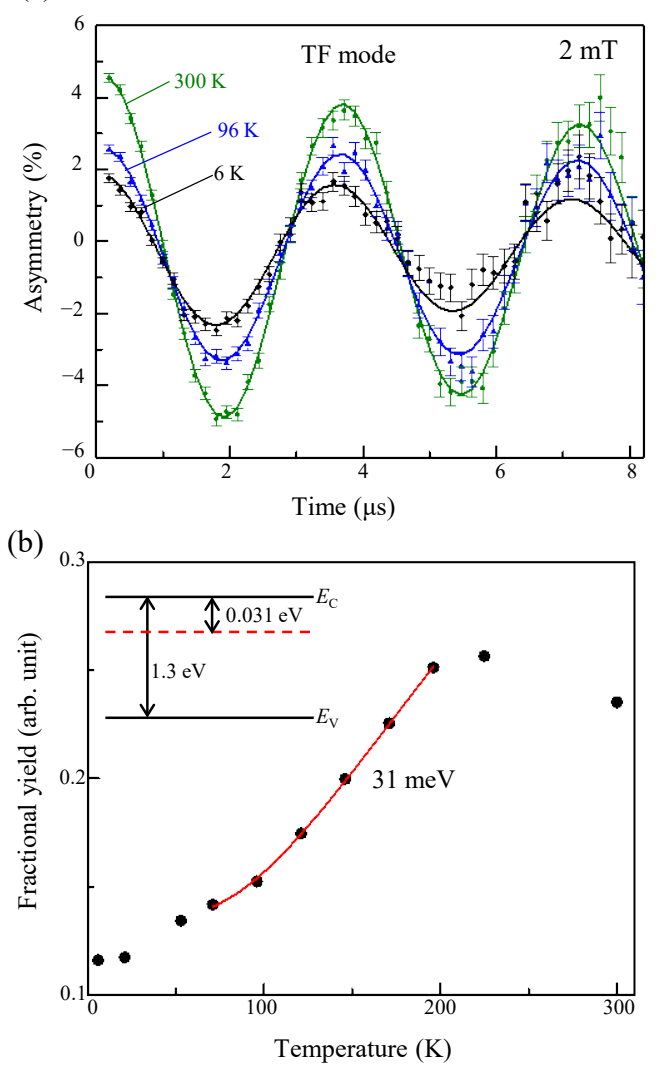

Fig. 3 


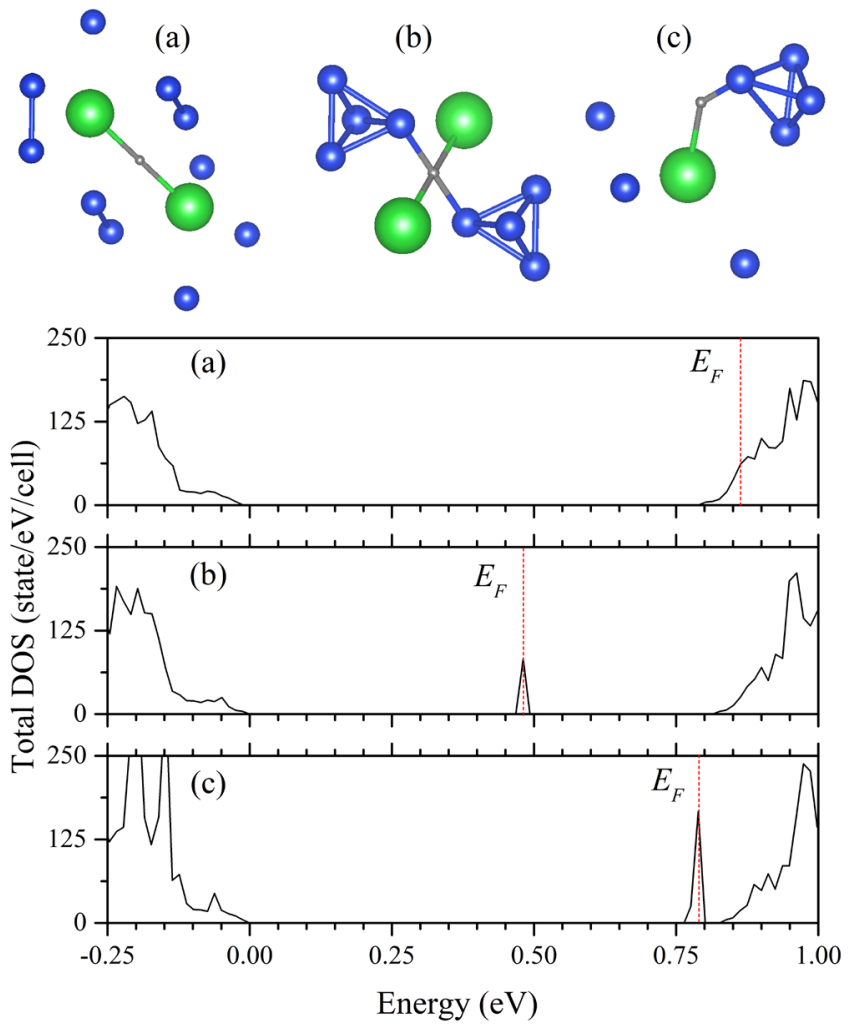

Fig. 4 

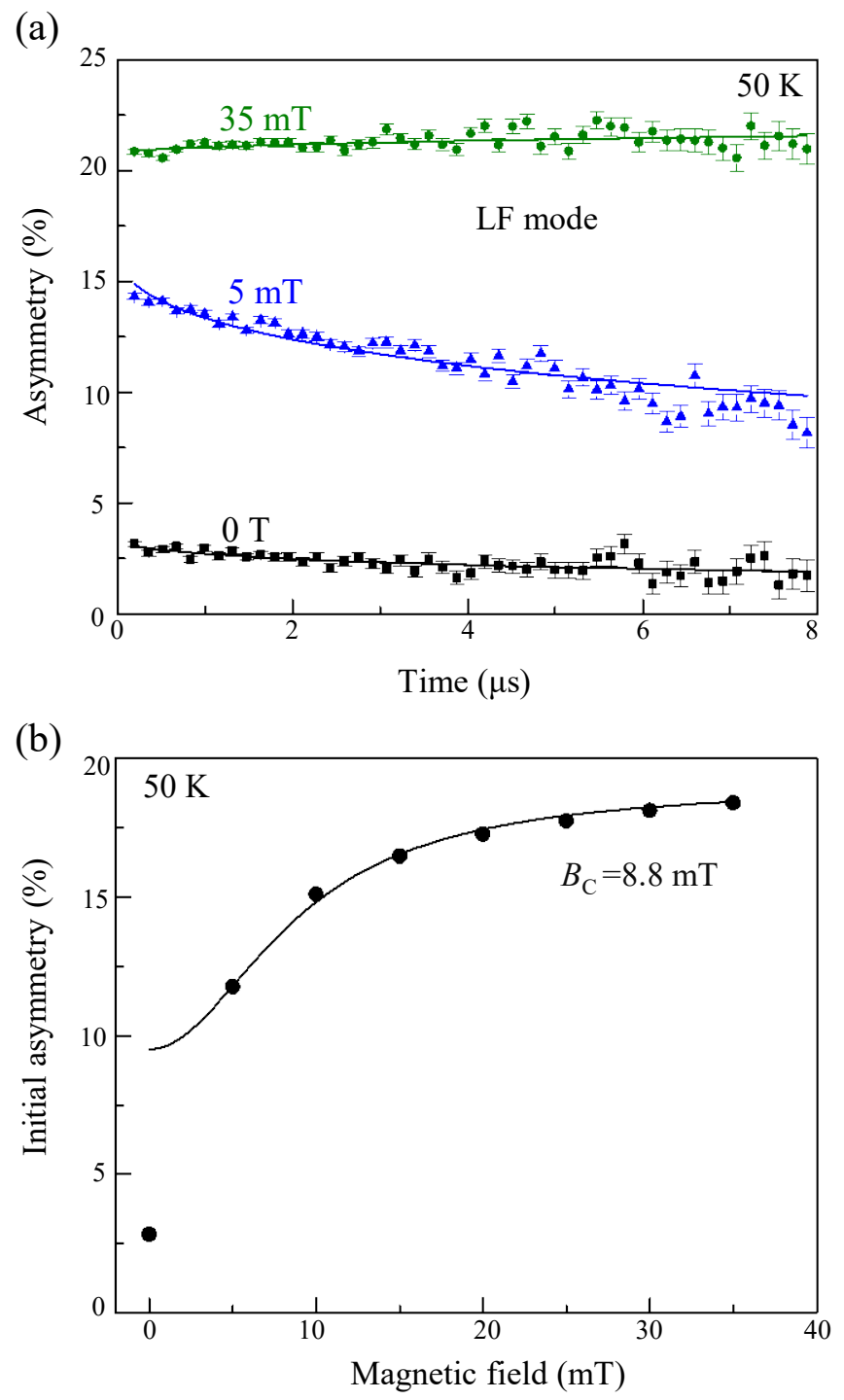

Fig. 5 